\title{
Targeting ryanodine receptors for anti-arrhythmic therapy
}

\author{
Mark D McCAULEY ${ }^{1,2}$, Xander H T WEHRENS ${ }^{1,2, *}$ \\ ${ }^{1}$ Department of Molecular Physiology and Biophysics; ${ }^{2}$ Department of Medicine (Cardiology), Baylor College of Medicine, Houston, TX, \\ USA
}

Antiarrhythmic drugs are a group of pharmaceuticals that suppress or prevent abnormal heart rhythms, which are often associated with substantial morbidity and mortality. Current antiarrhythmic drugs that typically target plasma membrane ion channels have limited clinical success and in some cases have been described as being pro-arrhythmic. However, recent studies suggest that pathological release of calcium $\left(\mathrm{Ca}^{2+}\right)$ from the sarcoplasmic reticulum via cardiac ryanodine receptors (RyR2) could represent a promising target for antiarrhythmic therapy. Diastolic SR $\mathrm{Ca}^{2+}$ release has been linked to arrhythmogenesis in both the inherited arrhythmia syndrome 'catecholaminergic polymorphic ventricular tachycardia' and acquired forms of heart disease (eg, atrial fibrillation, heart failure). Several classes of pharmaceuticals have been shown to reduce abnormal RyR2 activity and may confer protection against triggered arrhythmias through reduction of SR $\mathrm{Ca}^{2+}$ leak. In this review, we will evaluate the current pharmacological methods for stabilizing RyR2 and suggest treatment modalities based on current evidence of molecular mechanisms.

Keywords: arrhythmias; atrial fibrillation; calcium; heart failure; ryanodine receptor; sarcoplasmic reticulum

Acta Pharmacologica Sinica (2011) 32: 749-757; doi: 10.1038/aps.2011.44

\section{Introduction}

The cardiac ryanodine receptor (RyR2) is a homotetrameric $\mathrm{Ca}^{2+}$ release channel located in the sarcoplasmic reticulum (SR) membrane $\mathrm{e}^{[1,2]}$. During the normal cardiac cycle, plasma membrane depolarization initiates opening of L-type $\mathrm{Ca}^{2+}$ channels (LTCC), by which extracellular $\mathrm{Ca}^{2+}$ enters the cytoplasm. $\mathrm{Ca}^{2+}$ influx acts as a trigger that subsequently activates RyR2 channels, leading to a ten-fold greater release of SR $\mathrm{Ca}^{2+}$ into the cytoplasm. During systolic contraction of the heart, elevated cytoplasmic $\mathrm{Ca}^{2+}$ binds to troponin-C, allowing actin and myosin to interdigitate and cause sarcomere shortening, thus causing myocardial contraction. Diastolic relaxation occurs when cytoplasmic $\left[\mathrm{Ca}^{2+}\right]$ decreases as $\mathrm{Ca}^{2+}$ is extruded through the $\mathrm{Na}^{+} / \mathrm{Ca}^{2+}$-exchanger (NCX) or is actively pumped back into the SR through the sarco/endoplasmic reticulum $\mathrm{Ca}^{2+}$-ATPase $(\text { SERCA2a })^{[3]}$. Concomitantly, myocardial relaxation is directly associated with diastolic reduction in $\mathrm{Ca}^{2+}$ levels. Thus, physiologic control of $\mathrm{Ca}^{2+}$ release from the SR is necessary for timely contraction and relaxation during the cardiac cycle. Pathological "leak" of $\mathrm{Ca}^{2+}$ during diastole may be detrimental

\footnotetext{
* To whom correspondence should be addressed.

E-mail wehrens@bcm.edu

Received 2011-03-01 Accepted 2011-04-07
}

and lead to cardiac arrhythmias ${ }^{[4,5]}$.

There is now considerable evidence that abnormal RyR2mediated $\mathrm{Ca}^{2+}$ release from the SR can lead to both atrial ${ }^{[6,7]}$ and ventricular arrhythmias associated with sudden cardiac death $^{[8-10]}$. Increased SR $\mathrm{Ca}^{2+}$ release during diastole can lead to activation of the $\mathrm{Na}^{+} / \mathrm{Ca}^{2+}$-exchanger ${ }^{[11]}$, which in turn generates a transient inward current that can cause afterdepolarizations and triggered action potentials. These afterdepolarizations have been observed in humans and have been directly linked to arrhythmogenesis in animal models of arrhythmias $^{[12]}$.

Genetic susceptibility to cardiac arrhythmias may arise directly from genetic mutations in RyR2, such as in patients with catecholaminergic polymorphic ventricular tachycardia $(\mathrm{CPVT})^{[9,13]}$. Mutations in other proteins that bind to the poreforming subunits within the RyR2 macromolecular complex (eg, calsequestrin, junctophilin) also have been reported to confer genetic susceptibility to cardiac arrhythmias and/or cardiomyopathy ${ }^{[14,15]}$. These observations provide direct evidence that a perturbation in RyR2 function can facilitate the development of cardiac arrhythmias.

Additionally, acquired structural heart disease, for example heart failure or myocardial ischemia, has been shown to modify the post-translational regulation of RyR2 through 
nitrosylation, oxidation, and phosphorylation, which might also increase susceptibility to diastolic $\mathrm{Ca}^{2+}$ release and arrhythmias ${ }^{[16-20]}$. Given that there are many excellent reviews on strategies to modify intracellular signaling to reduce RyR2 activation ${ }^{[21,22]}$, we will restrict the scope of this review mainly to pharmacological strategies to stabilize RyR2 directly to reduce arrhythmic potential.

Because RyR2 also plays an important role during excitation-contraction coupling, it is important that antiarrhythmic compounds targeting the RyR2 channel complex will not interfere with systolic SR $\mathrm{Ca}^{2+}$ release. At the same time, inhibition of diastolic SR $\mathrm{Ca}^{2+}$ release is a desirable feature of compounds that could prevent arrhythmias ${ }^{[22]}$. RyR2 activity can be modulated by numerous natural and pharmacological compounds, as reviewed elsewhere in more detail ${ }^{[22-24]}$. These compounds may modulate RyR2 in various ways, including by modulating channel gating, ion channel translocation, RyR2 subunit composition, or posttranslational modifications. Some of these compounds have emerged as strong candidates for antiarrhythmic drugs, and will be discussed in more detail below.

\section{Dantrolene}

Dantrolene sodium is a hydrantoin derivative that was initially described as a muscle relaxant ${ }^{[25]}$, but later found to be a potent therapeutic agent for patients suffering from the rare life-threatening condition known as malignant hyperthermia $(\mathrm{MH})^{[26]}$. Patients susceptible to $\mathrm{MH}$ typically have inherited mutations in the type 1 ryanodine receptor (RyR1) primarily found in skeletal muscle ${ }^{[27]}$. Exposure to inhaled halogenated anesthetics during surgery can trigger massive RyR1-mediated $\mathrm{Ca}^{2+}$ release associated with muscle breakdown, elevation of serum creatinine kinase (CK), hypotension, hyperthermia, and tachycardia, which often results in intraoperative death ${ }^{[28]}$. Dantrolene has been shown to directly bind to the N-terminus of RyR1 and to prevent SR $\mathrm{Ca}^{2+}$ leak in skeletal muscle, thereby improving clinical outcomes ${ }^{[29]}$. Dantrolene is believed to stabilize interdomain interactions between the $\mathrm{N}$-terminal and central domains of RyR $1^{[30]}$ and RyR2 $2^{[31]}$, although the effects of dantrole on single RyR channels remains controversial ${ }^{[32]}$.

Given that dantrolene improves the stability of both RyR1 and RyR2, dantrolene has become a molecule of interest for preventing cardiac arrhythmias. Dantrolene has previously been described as an inhibitor of arrhythmias in animal models of ischemia-reperfusion ${ }^{[33-35]}$. More recently, dantrolene was demonstrated to inhibit catecholaminergic polymorphic ventricular tachycardia in a knock-in mouse model heterozygous for mutation R2474S in RyR2 ${ }^{[36]}$ (Figure 1). Dantrolene was shown to suppress isoproterenol-induced spontaneous $\mathrm{SR} \mathrm{Ca}^{2+}$ releases (ie, sparks) in intact myocytes isolated from RyR2-R2474A/+ mice. The mechanisms by which dantrolene prevented CPVT was attributed to stabilization of mutant RyR2 channels, and possibly also by preventing the PKAinduced reduction in calmodulin binding to RyR2 $2^{[37]}$. In this study, dantrolene did not exert any appreciable effects on cardiac function in hearts of wild-type mice. However, dan- trolene did correct defective interdomain interactions within RyR2 isolated from dogs with heart failure, associated with suppression of delayed afterdepolarizations ${ }^{[31]}$

In other studies, dantrolene has been described to improve cardiac contractility in failing hearts, which may contribute to its role in reducing arrhythmias in structural heart disease. Congestive heart failure ( $\mathrm{CHF}$ ) has been associated with a negative force-frequency relationship (Bowditch effect) in failing myocardium. Dantrolene has been shown to ameliorate the negative force-frequency relationship in explanted failing myocardial muscle strips by improving inotropic response to isoproterenol $^{[38]}$. This improved contractility was not associated with overall changes in cytoplasmic $\left[\mathrm{Ca}^{2+}\right]$, and was postulated to be associated with improvement of diastolic $\mathrm{Ca}^{2+}$ release. Further evidence for reduction of $\mathrm{Ca}^{2+}$ release events in improved contractility was shown by Kobayashi et al ${ }^{[31]}$, who examined the effects of dantrolene on cardiac function on failing hearts. Dantrolene was shown to directly bind near the N-terminal domain in RyR2 at amino acids 601-620, a site critical for N-terminal and central domain interactions. Whereas unzipping of N-terminal and central domains was associated with spontaneous SR $\mathrm{Ca}^{2+}$ leak, dantrolene suppressed both unzipping and SR $\mathrm{Ca}^{2+}$ leak (sparks) and ultimately delayed afterdepolarizations, which are common in heart failure. Additionally, dantrolene restores calmodulin (CaM) binding to RyR2, which is usually attenuated in heart failure ${ }^{[39]}$. Thus, dantrolene appears to be a promising molecule to treat arrhythmias in patients with CPVT and may attenuate cardiac $\mathrm{Ca}^{2+}$ handling dysfunction associated with heart failure.

\section{1,4-Benzothiazepines}

The benzothiazepine derivative JTV519 (4-[3(1-(4-benzyl) piperidinyl)propionyl]-7-methoxy-2,2,4,5-tetrahydro-1,4benzothiazepine; also known as K201) was first identified as a compound able to suppress intracellular $\mathrm{Ca}^{2+}$ overload associated with cardiac cell death ${ }^{[40]}$. The drug has been reported to have antiarrhythmic effects in a canine model of atrial fibrillation due to sterile pericarditis ${ }^{[41]}$ and Langendorff-perfused rat hearts subjected to ischemia-reperfusion ${ }^{[42,43]}$. JTV519 interacts with annexin- $\mathrm{V}$ and at higher doses inhibits various voltage-gated ion channels in the heart ${ }^{[44,45]}$. Subsequently, it has become clear that RyR2 represents an important target of JTV519 ${ }^{[46,47]}$.

JTV519 was described to normalize RyR2 gating in dogs with tachycardia-induced heart failure ${ }^{[48]}$. In this study, Kohno et al ${ }^{[48]}$ demonstrated that JTV519 reversed the SR Ca ${ }^{2+}$ release defects indicative of RyR2 dysfunction. The concept of RyR2 stabilization by FKBP12.6 was further supported by the findings that JTV519 treatment of dogs with pacing-induced heart failure increased the amount of FKBP12.6 immunoprecipitated with RyR2 ${ }^{[46]}$. Moreover, JTV519 was shown to prevent lethal ventricular arrhythmias in mice haploinsufficient for FKBP12.6 by increasing FKBP12.6 binding to RyR2 ${ }^{[47]}$ (Figure 2). The lack of efficacy of JTV519 in FKBP12.6 deficient mice suggests that FKBP12.6 binding to RyR2 is associated with the therapeutic effects of this compound ${ }^{[47]}$. On the other hand, 
A
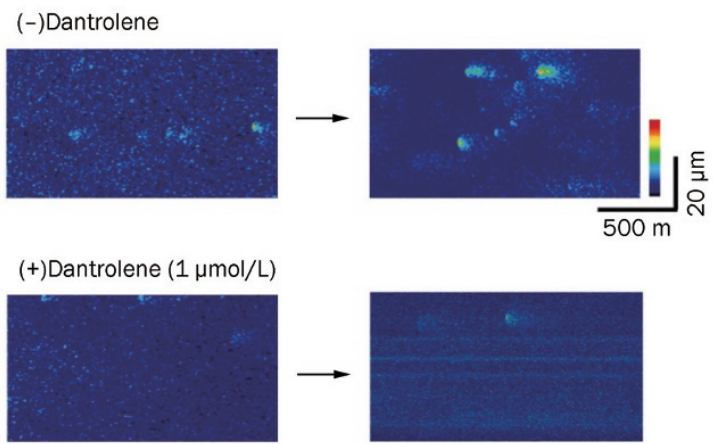

Baseline

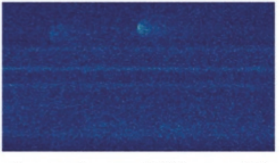

Isoproterenol $10 \mathrm{nmol} / \mathrm{L}$
B

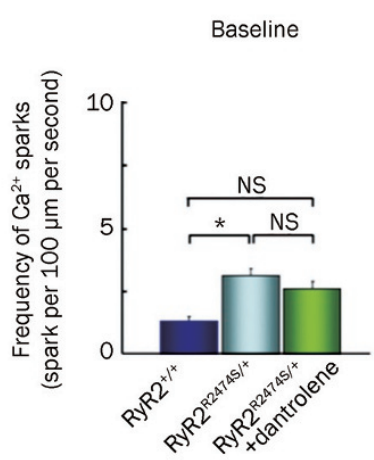

Isoproterenol $10 \mathrm{nmol} / \mathrm{L}$

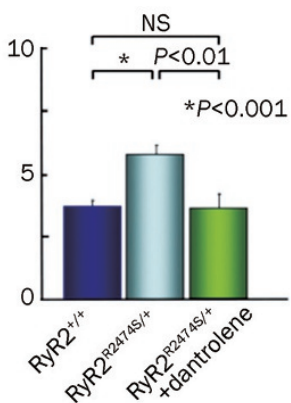

D
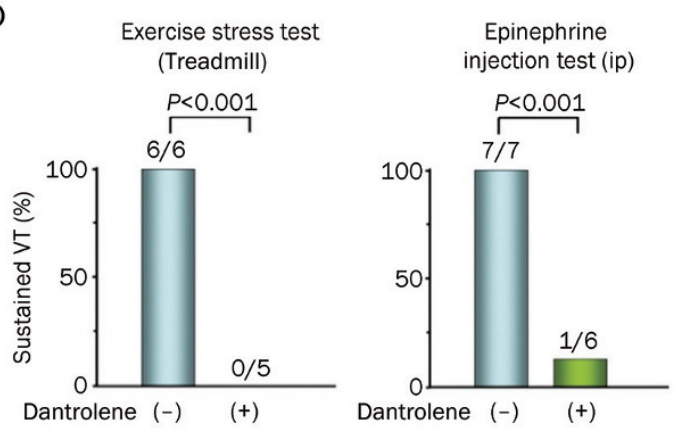

Figure 1. Dantrolene inhibits catecholaminergic polymorphic ventricular tachycardia in mice. (A) Representative images of Ca ${ }^{2+}$ sparks in cardiomyocytes isolated from heterozygous RyR2-R2474S/+ knock-in mice, showing that dantrolene reduces Ca ${ }^{2+}$ spark frequency following isoproterenol exposure. (B) Bar graph showing that dantrolene suppresses abnormal $\mathrm{Ca}^{2+}$ spark frequency in RyR2-R2474S/+ mutant mice after isoproterenol exposure. (C) Telemetric ECG recordings reveal exercise-induced ventricular tachycardia in a RyR2-R2474S/+ mouse, which was suppressed by dantrolene. (D) Bar graphs revealing that dantrolene suppresses the incidence of exercise or epinephrine induced ventricular tachycardia (VT) in RyR2R2474S/+ mice. Adapted from Kobayashi et al ${ }^{[36]}$.

normalizing FKBP12.6 levels within the RyR2 macromolecular complex stabilizes the closed state of the channel, thereby preventing aberrant openings during diastole ${ }^{[5]}$. Follow-up in vitro studies of human RyR2 mutations found in patients with CPVT (P2328S, Q4201R, and V4653F) showed that JTV519 can also normalize mutant channel gating as evidenced by single channel recordings ${ }^{[49]}$.

There has been some controversy whether the antiarrhythmic effects of JTV519 require modification of RyR2-FKBP12.6 interactions ${ }^{[50]}$. In a mouse model of CPVT caused by the R4496C mutation in RyR2, it was shown that this mutation did not alter FKBP12.6 binding affinity for RyR2. Moreover, JTV519 did not prevent delayed afterdepolarizations in myocytes isolated from heterozygous RyR2-R4496C/+ mice ${ }^{[51]}$. However, subsequent studies by other groups have shown that JTV519 did reduce the occurrence of spontaneous action potentials in ouabain-treated WT and RyR2-R4496/+ mouse myocytes, presumably independent of FKBP12.6 $6^{[52]}$. Additionally, in vitro studies in HEK293 cells suggest that JTV519 suppresses store-overload induced $\mathrm{Ca}^{2+}$ release independently of FKBP12.6 binding, though the relevance of these observations have yet to be determined in vivo ${ }^{[50]}$. Also, Yamamoto et al ${ }^{[53]}$ reported that JTV519 directly bound to RyR2 between amino acids 2114 and 2149, and that JTV519 can normalize defective interdomain interactions associated with RyR2 dysfunction.

Recently S107, a new 1,4-benzothiazepine similar to JTV519, has been found to prevent ventricular arrhythmias in a CPVT mouse model heterozygous for mutation R2474S in RyR2 ${ }^{[54]}$. In contrast to JTV519, S107 has been reported to lack off-target activity for ion channels other than RyR2 at concentrations up to $10 \mathrm{mmol} / \mathrm{L}^{[54,55]}$. S107 provided protection against epinephrine-induced ventricular tachycardias caused by abnormal SR Ca ${ }^{2+}$ leak in RyR2-R2474S/+ mice ${ }^{[54]}$. Further, S107 was recently shown to be effective at preventing ventricular arrhythmias in the $m d x$ mouse model of muscular dystrophy ${ }^{[56]}$. Thus, 1,4-benzothiazepine derivatives JTV519 and S107 hold promise as RyR2-stabilizing molecules that could reduce the risk of arrhythmias ${ }^{[57]}$.

\section{Flecainide}

Flecainide is a trifluoroethoxybenzamide that was discovered to be a potent antiarrhythmic agent in $1977^{[58]}$. Flecainide initially showed promise as an antiarrhythmic agent against both ventricular $^{[59]}$ and atrial arrhythmias ${ }^{[60]}$. Because a predomi- 
A
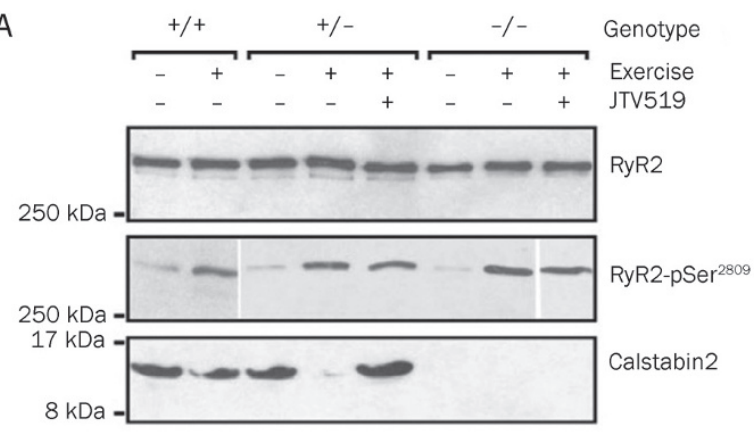

C

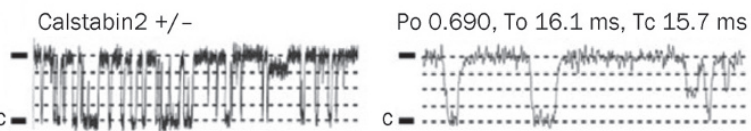

Calstabin2 +/-, JTV519

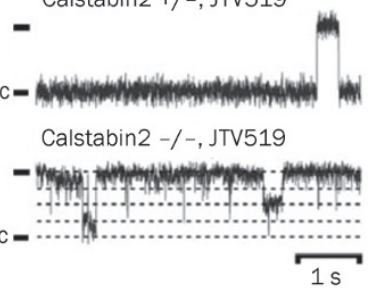

Po 0.005, To 17.2 ms, Tc 3654 ms

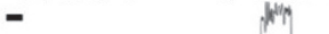

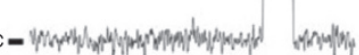

Po 0.965, To $14.1 \mathrm{~ms}$, Tc $3.27 \mathrm{~ms}$

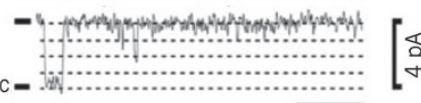

B
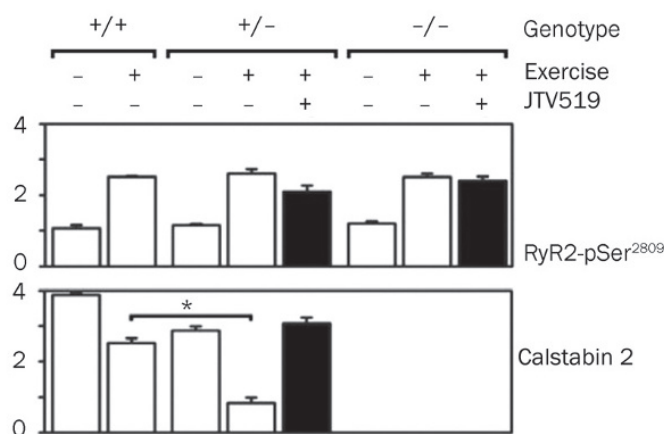

E

ECG following exercise + epinephrine

$(\mathrm{mV})$

(1)

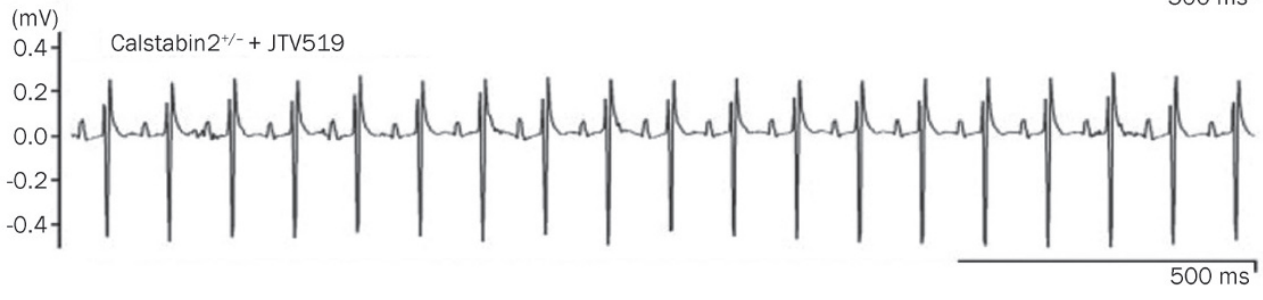

F
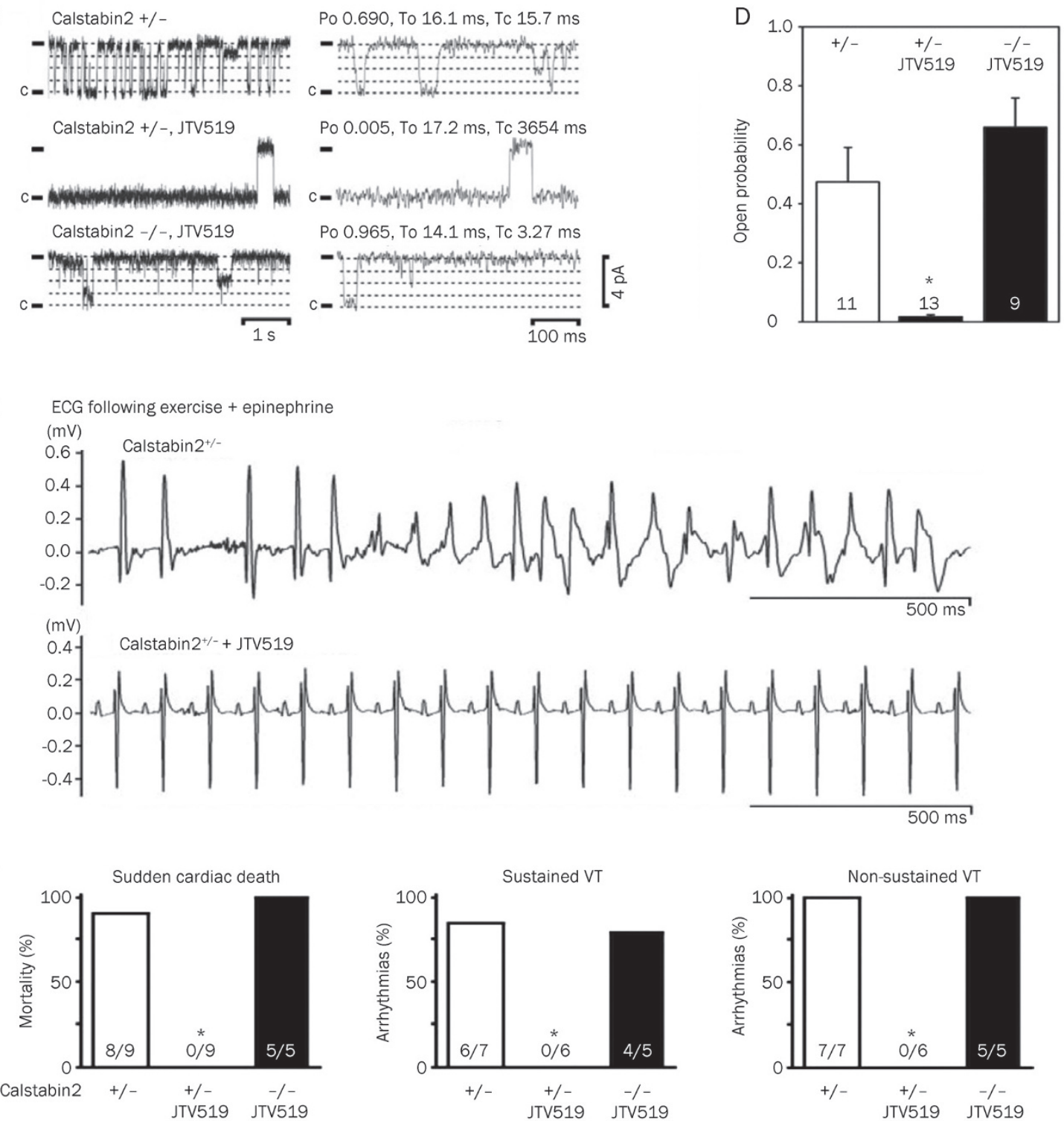

Figure 2. Anti-arrhythmic effects of 1,4-benzothiazepine JTV-519 in FKBP12.6 ${ }^{+/-}$mice. (A-B) Representative immunoblots and quantifications for RyR2, RyR2-pSer ${ }^{2809}$ (PKA phosphorylation site on RyR2), and calstabin2 (FKBP12.6) from wildtype (WT), calstabin2 ${ }^{+/-}$heterozygous, and calstabin2 ${ }^{-/-}$ (FKBP12.6 $6^{-/}$) knockout mice. Whereas exercise increased PKA phosphorylation of RyR2 and decreased calstabin2 (FKBP12.6) binding to RyR2, JTV519 prevented calstabin2 dissociation. (C-D) Representative single channel recordings in planar lipid bilayers showing that JVT519 reduced the open probability of RyR2 isolated from calstabin $2^{+/-}$but not calstabin $2^{-/-}$mice, consistent with calstabin2 (FKBP12.6) being required for the therapeutic effects of JTV519. (E-F) ECG tracings showing that JTV-519 reduces ventricular arrhythmias in calstabin2 ${ }^{+/-}$but not calstabin2 ${ }^{-/-}$mice. ${ }^{*} P<0.05$. Adapted from Wehrens et $a l^{[47]}$. 
nant mechanism of action on inactivation of voltage-gated $\mathrm{Na}^{+}$ channels, it was classified as a type IC anti-arrhythmic drug. However, clinical trial results indicated that in patients with structural heart disease susceptible to ventricular arrhythmias, flecainide might in fact be pro-arrhythmogenic ${ }^{[61,62]}$.

Recently, there has been a resurgence of enthusiasm for the use of flecainide in a select group of CPVT patients with genetic predisposition to ventricular arrhythmias and SCD. Watanabe et $a l^{[63]}$ found that flecainide inhibited the RyR2 channel by reducing the duration of RyR2 channel openings without affecting closed channel duration. Flecainide reduced $\mathrm{SR} \mathrm{Ca}{ }^{2+}$ release events and triggered arrhythmic beats in a calsequestrin deficient (Casq2 $2^{--}$) mouse model of CPVT ${ }^{[63]}$ (Figure 3). Moreover, it was shown that flecainide significantly reduced the incidence of exercise-induced arrhythmias in patients with mutations in $C A S Q 2^{[63]}$. Follow-up studies from the same group showed that flecainide reduced $\mathrm{Ca}^{2+}$ spark mass but increased spark frequency, resulting in a net neutral effect on SR Ca ${ }^{2+}$ leak and SR $\mathrm{Ca}^{2+}$ content $^{[64]}$. This finding is distinct from the reported mechanism of tetracaine, another RyR2 channel blocking agent, which reduces $\mathrm{Ca}^{2+}$ sparks and SR leak, thereby increasing SR $\mathrm{Ca}^{2+}$ content. Therefore, it was concluded that flecainide promoted block of the RyR2 open state, reducing the "probability of saltatory wave propagation between adjacent $\mathrm{Ca}^{2+}$ release units" ${ }^{\text {[64] }}$.
Other groups have applied these findings to further delineate the mechanism of arrhythmogenesis in another mouse model of CPVT. Knock-in mice heterozygous for mutation R4496/+ in RyR2 were crossed with Cntn2-EGFP transgenic mice expressing a fluorescent marker for the cardiac conduction system ${ }^{[65]}$. Whereas tetracaine reduced spontaneous SR $\mathrm{Ca}^{2+}$ release events in ventricular myocytes and Purkinje cells equally, flecainide more specifically targeted mutant RyR2 in Purkinje cells, implicating the Purkinje conduction system as a potent mediator of ventricular arrhythmias in CPVT. Thus, flecainide may have a unique role in the prevention and suppression of ventricular arrhythmias in patients with genetically inherited CPVT.

\section{Modulation of RyR2 posttranslational modification}

In addition to inherited mutations, RyR2 channel function may also be perturbed due to acquired changes in, for example, channel posttranslational modulation ${ }^{[2]}$. Xu et al ${ }^{[20]}$ demonstrated that increased S-nitrosylation leads to enhanced RyR2 activity and promotes SR $\mathrm{Ca}^{2+}$ release. Increased S-nitrosylation of RyR2 has been associated with cardiac arrhythmias in a mouse model of Duchenne's muscular dystrophy, and inhibition with $\mathrm{S} 107$ (see above) was shown to normalize both RyR1 and RyR2 function and prevent arrhythmias ${ }^{[56,66]}$. In contrast, Gonzalez et al ${ }^{[17]}$ demonstrated that decreased rather
A $0 \mu \mathrm{mol} / \mathrm{L}$ flecainide

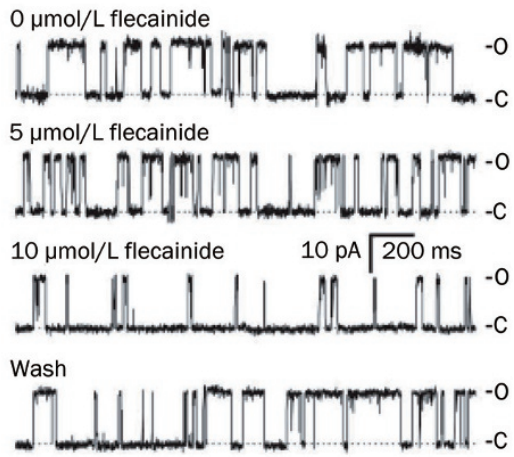

B

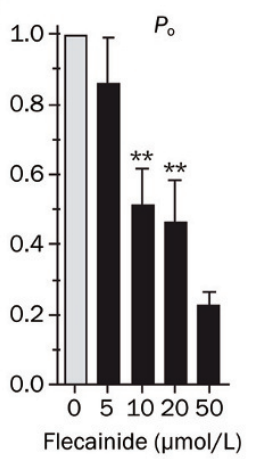

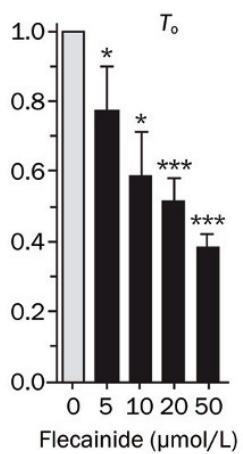

$T_{0}$

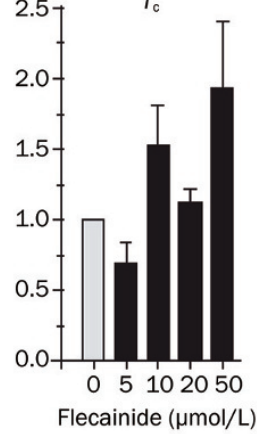

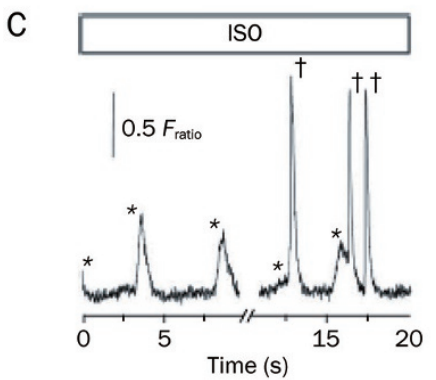
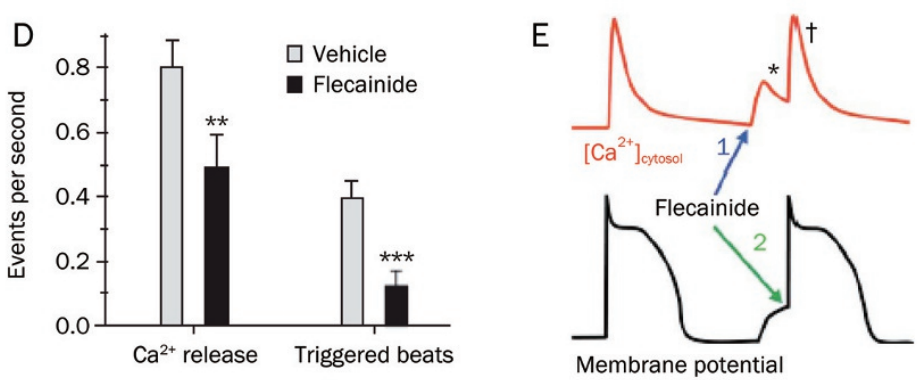

Figure 3. Prevention of triggered arrhythmias by flecainide. (A-B) Concentration-dependent effects of flecainide on single sheep RyR2 channels in lipid bilayers. Flecainide decreases open probability (Po) and mean open time (To), and does not significantly alter the mean closed time (Tc) of RyR2. $* P<0.02, * * P<0.01$ and $* * * P<0.001$. (C-D) Effects of flecainide on isoproterenol (ISO) stimulated calsequestrin-deficient cardiomyocytes. Whereas ISO evoked spontaneous SR $\mathrm{Ca}^{2+}$ release events $(*)$, flecainide reduced the number of $\mathrm{Ca}^{2+}$ releases and triggered beats $(\dagger) . * * P=0.0078$ and $* * * P$ $<0.001$. (E) Cartoon illustrating dual effects of flecainide action on SR $\mathrm{Ca}^{2+}$ release (red tracing) and inhibition of premature beats triggered by delayed after depolarization (black tracing). Adapted from Watanabe et al ${ }^{[63]}$. 
than increased S-nitrosylation of RyR2 might promote SR $\mathrm{Ca}^{2+}$ leak and arrhythmogenesis. One explanation of this apparent paradox relates to nitroso-redox imbance, a condition in which excess formation of reactive oxygen species (ROS) can modify the same thiols that are also target of $S$-nitrosylation ${ }^{[67]}$. Indeed, Gonzalez et al ${ }^{[67]}$ reported evidence for increased oxidation of RyR2 associated with an increased tendency towards $\mathrm{SR} \mathrm{Ca}^{2+}$ leak in rats with heart failure. In this particular study, increased oxidative stress was primarily the result of enhanced xanthine oxidase $(\mathrm{XO})$ activity. Pharmacological inhibition of $\mathrm{XO}$ restored both the nitroso-redox imbalance and intracellular $\mathrm{Ca}^{2+}$ release defects in these rats with heart failure ${ }^{[67]}$. Moreover, Niggli's group showed that anti-oxidants (ie, MPG and Mn-cpx3) normalized abnormal RyR sensitivity and hypersensitive E-C coupling in dystrophic cardiomyocytes ${ }^{[68]}$.

Increased oxidative stress might also promote activation of $\mathrm{Ca}^{2+} /$ calmodulin-dependent protein kinase, which can phosphorylate RyR2 along with other $\mathrm{Ca}^{2+}$ handling proteins, and increase the propensity towards cardiac arrhythmias ${ }^{[69]}$. We have previously shown that CaMKII phosphorylation of RyR2 leads to increased channel open probability ${ }^{[19]}$. Recently, we demonstrated that constitutive phosphorylation of RyR2 by CaMKII in RyR2-S2814D knock-in mice promoted abnormal $\mathrm{SR} \mathrm{Ca}^{2+}$ release events associated with ectopic activity and ventricular arrhythmias ${ }^{[10]}$. On the other hand, genetic inhibition of RyR2 phosphorylation at S2814 in RyR2-S2814A knockin mice conferred protection against ventricular arrhythmias in mice with heart failure induced by transverse aortic banding $^{[10]}$. These studies suggest that inhibition of RyR2 phosphorylation by CaMKII might provide a very specific way of preventing ventricular and also atrial arrhythmias ${ }^{[6]}$. Moreover, pharmacological inhibition of the enzyme CaMKII itself might also provide protection against arrhythmias ${ }^{[6,70,71]}$.

RyR2 is also regulated by protein kinase A (PKA) phosphorylation, and increased PKA phosphorylation of RyR2 has been observed in patients with atrial fibrillation ${ }^{[7]}$. Shan et $a l^{[57]}$ demonstrated that mice in which RyR2 was constitutively by PKA (RyR2-S2808D knock-in mice) exhibited an increased open probability, more calcium sparks, and increased SR $\mathrm{Ca}^{2+}$ leak. Inhibition of PKA phosphorylation of RyR2 in RyR2S2808A mice was shown to protect against catecholamineinduced ventricular arrhythmias ${ }^{[72]}$. Although there are currently no drugs that specifically reduce RyR2 phosphorylation, beta blockers such as carvedilol have been shown to reduce RyR2 phosphorylation and thereby RyR2 open probability in patients with atrial fibrillation ${ }^{[73]}$. In addition, some beta blockers such as carvedilol also have antioxidant properties in addition to beta-adrenergic blockade, and may be useful in prolongation of arrhythmia-free survival in patients with congestive heart failure versus beta blockers lacking anti-oxidant properties ${ }^{[74]}$. Clearly, further pharmacological studies would be needed to determine whether modulating post-translational modifications of RyR2 represents a suitable anti-arrhythmic strategy.

\section{Conclusions}

Cardiac arrhythmias is a potentially life-threatening complication of genetic and structural heart disease. Recent insights into excitation-contraction coupling have implicated release of SR $\mathrm{Ca}^{2+}$ through RyR2 as a key mechanism for the initiation and maintenance of both atrial and ventricular arrhythmias. RyR2-mediated release of SR $\mathrm{Ca}^{2+}$ is a tightly regulated process that involves discrete release of $\mathrm{Ca}^{2+}$ during systole, and cessation of $\mathrm{Ca}^{2+}$ release during diastole. For timely rhythmic release of $\mathrm{Ca}^{2+}$ from RyR2, the channel must succinctly open in response to cytoplasmic $\mathrm{Ca}^{2+}$ flux, but remain closed during diastolic SR $\mathrm{Ca}^{2+}$ filling. Destabilization of RyR2 may occur as a result of genetic mutations (ie, CPVT) or acquired (eg, oxidation, nitrosylation, phosphorylation) modifications, resulting in pathologic diastolic $\mathrm{Ca}^{2+}$ release, and subsequent arrhythmias.

Given the prominent role of RyR2 in SR $\mathrm{Ca}^{2+}$ release, pharmacological strategies to modulate RyR2 stability and gating have shown great promise as a therapy for cardiac arrhythmias. Several drugs targeting RyR2, such as benzothiazepine derivatives and flecainide, bind RyR2 directly and reduce the open probability of RyR2, thereby reducing pathological SR $\mathrm{Ca}^{2+}$ "leak". As benzothiazepines and flecainide have an additional role in blockade of voltage-gated sodium channels and delayed rectifier potassium channels, there may be an additive effect on anti-arrhythmic action, however further studies are necessary to evaluate whether this may occur independently of RyR2 blockade or enhancement of RyR2-FKBP12.6 binding. Additionally, dantrolene has been shown to bind directly to RyR2 and stabilize inter-domain regions, although the effects on RyR2 open probability are still controversial. Each of these drugs has a unique mechanism of action, as dantrolene stabilizes N-terminal and central domain interactions, benzothiazepines increase FKBP12.6 (calstabin2) binding to RyR2 (among other mechanisms), and flecainide blocks the open state of the channel. These drugs have proven particularly helpful in CPVT, in which stabilization of RyR2 reduces diastolic SR $\mathrm{Ca}^{2+}$ leak, and therefore reduces delayed afterdepolarizations.

In patients with structural heart disease, such as in congestive heart failure, acquired alterations in RyR2 function occur primarily due to post-translational modification of the channel. There is extensive evidence that hyperphosphorylation of RyR2 in heart failure also promotes the occurrence of SR Ca ${ }^{2+}$ leak ${ }^{[10,18,72,75]}$. By genetic or pharmacological blockade of RyR2 phosphorylation at CaMKII or PKA site, animal models have shown that effective arrhythmia prophylaxis is possible, especially under catecholaminergic conditions or after stress exercise. Recent insights also indicate that adverse redox remodeling of RyR2 may predispose to cardiac arrhythmias. Emerging data suggest that certain beta-adrenergic blocking agents, such as carvedilol, may also exert a redox-stabilizing effect on RyR2, which may potentially increase survival in patients with acquired heart disease. Ultimately, these insights will guide the design of future studies in human patients, whereby stabilization of the RyR2 channel might lead to improved outcomes in morbidity and mortality. 


\section{Acknowledgements}

Dr McCauley is supported by NHLBI training grant 5T32HL066991-07. Dr WEHRENS is a WM Keck Foundation Distinguished Young Scholar in Medical Research and is supported by NHLBI grants R01-HL089598 and R01-HL091947, and Muscular Dystrophy Association grant 186531. This work was also supported in part by the Fondation Leducq Alliance for CaMKII Signaling in Heart.

\section{References}

1 Bers DM. Cardiac excitation-contraction coupling. Nature 2002; 415: 198-205.

2 Wehrens XH, Lehnart SE, Marks AR. Intracellular calcium release and cardiac disease. Annu Rev Physiol 2005; 67: 69-98.

3 Dobrev D, Wehrens XH. Calmodulin kinase II, sarcoplasmic reticulum $\mathrm{Ca}^{2+}$ leak, and atrial fibrillation. Trends Cardiovasc Med 2010; 20 : 30-4.

4 Laitinen PJ, Brown KM, Piippo K, Swan H, Devaney JM, Brahmbhatt $\mathrm{B}$, et al. Mutations of the cardiac ryanodine receptor (RyR2) gene in familial polymorphic ventricular tachycardia. Circulation 2001; 103: 485-90.

5 Wehrens XH, Lehnart SE, Huang F, Vest JA, Reiken SR, Mohler PJ, et al. FKBP12.6 deficiency and defective calcium release channel (ryanodine receptor) function linked to exercise-induced sudden cardiac death. Cell 2003; 113: 829-40.

6 Chelu MG, Sarma S, Sood S, Wang S, van Oort RJ, Skapura DG, et al. Calmodulin kinase II-mediated sarcoplasmic reticulum $\mathrm{Ca}^{2+}$ leak promotes atrial fibrillation in mice. J Clin Invest 2009; 119: 1940-51.

7 Vest JA, Wehrens XH, Reiken SR, Lehnart SE, Dobrev D, Chandra P, et al. Defective cardiac ryanodine receptor regulation during atrial fibrillation. Circulation 2005; 111: 2025-32.

8 Mathur N, Sood S, Wang S, van Oort RJ, Sarma S, Li N, et al. Sudden infant death syndrome in mice with an inherited mutation in RyR2. Circ Arrhythm Electrophysiol 2009; 2: 677-85.

9 Priori SG, Napolitano C, Tiso N, Memmi M, Vignati G, Bloise R, et al. Mutations in the cardiac ryanodine receptor gene (hRyR2) underlie catecholaminergic polymorphic ventricular tachycardia. Circulation 2001; 103: 196-200.

10 van Oort RJ, McCauley MD, Dixit SS, Pereira L, Yang Y, Respress JL, et al. Ryanodine receptor phosphorylation by calcium/calmodulindependent protein kinase II promotes life-threatening ventricular arrhythmias in mice with heart failure. Circulation 2010; 122: 266979.

11 Lehnart SE, Terrenoire C, Reiken S, Wehrens XH, Song LS, Tillman EJ, et al. Stabilization of cardiac ryanodine receptor prevents intracellular calcium leak and arrhythmias. Proc Natl Acad Sci U S A 2006; 103 7906-10.

12 Paavola J, Viitasalo M, Laitinen-Forsblom PJ, Pasternack M, Swan H, Tikkanen I, et al. Mutant ryanodine receptors in catecholaminergic polymorphic ventricular tachycardia generate delayed afterdepolarizations due to increased propensity to $\mathrm{Ca}^{2+}$ waves. Eur Heart J 2007; 28: $1135-42$

13 McCauley MD, Wehrens XH. Animal models of arrhythmogenic cardiomyopathy. Dis Model Mech 2009; 2: 563-70.

14 Landstrom AP, Weisleder N, Batalden KB, Bos JM, Tester DJ, Ommen $\mathrm{SR}$, et al. Mutations in JPH2-encoded junctophilin-2 associated with hypertrophic cardiomyopathy in humans. J Mol Cell Cardiol 2007; 42: 1026-35.

15 Song L, Alcalai R, Arad M, Wolf CM, Toka O, Conner DA, et al. Calsequestrin 2 (CASQ2) mutations increase expression of calreticulin and ryanodine receptors, causing catecholaminergic polymorphic ventricular tachycardia. J Clin Invest 2007; 117: 1814-23.

16 Belevych AE, Terentyev D, Viatchenko-Karpinski S, Terentyeva $\mathrm{R}$, Sridhar A, Nishijima $\mathrm{Y}$, et al. Redox modification of ryanodine receptors underlies calcium alternans in a canine model of sudden cardiac death. Cardiovasc Res 2009; 84: 387-95.

17 Gonzalez DR, Beigi F, Treuer AV, Hare JM. Deficient ryanodine receptor S-nitrosylation increases sarcoplasmic reticulum calcium leak and arrhythmogenesis in cardiomyocytes. Proc Natl Acad Sci U S A 2007; 104: 20612-7.

18 Marx SO, Reiken S, Hisamatsu Y, Jayaraman T, Burkhoff D, Rosemblit $\mathrm{N}$, et al. PKA phosphorylation dissociates FKBP12.6 from the calcium release channel (ryanodine receptor): defective regulation in failing hearts. Cell 2000; 101: 365-76.

19 Wehrens XH, Lehnart SE, Reiken SR, Marks AR. $\mathrm{Ca}^{2+} /$ calmodulindependent protein kinase II phosphorylation regulates the cardiac ryanodine receptor. Circ Res 2004; 94: e61-70.

$20 \mathrm{Xu} \mathrm{L}, \mathrm{Eu}$ JP, Meissner G, Stamler JS. Activation of the cardiac calcium release channel (ryanodine receptor) by poly-S-nitrosylation. Science 1998; 279: 234-7.

21 Anderson ME, Higgins LS, Schulman H. Disease mechanisms and emerging therapies: protein kinases and their inhibitors in myocardial disease. Nat Clin Pract Cardiovasc Med 2006; 3: 437-45.

22 Santonastasi M, Wehrens XH. Ryanodine receptors as pharmacological targets for heart disease. Acta Pharmacol Sin 2007; 28 : 937-44.

23 Dulhunty AF, Beard NA, Pouliquin P, Casarotto MG. Agonists and antagonists of the cardiac ryanodine receptor: potential therapeutic agents? Pharmacol Ther 2007; 113: 247-63.

24 Wehrens XH, Lehnart SE, Marks AR. Ryanodine receptor-targeted anti-arrhythmic therapy. Ann N Y Acad Sci 2005; 1047: 366-75.

25 Snyder HR Jr, Davis CS, Bickerton RK, Halliday RP. 1-[(5-arylfurfurylidene)amino]hydantoins. A new class of muscle relaxants. J Med Chem 1967; 10: 807-10.

26 Harrison GG. Control of the malignant hyperpyrexic syndrome in MHS swine by dantrolene sodium. Br J Anaesth 1975; 47: 62-5.

27 Durham WJ, Aracena-Parks P, Long C, Rossi AE, Goonasekera SA, Boncompagni S, et al. RyR1 S-nitrosylation underlies environmental heat stroke and sudden death in Y522S RyR1 knockin mice. Cell 2008; 133: 53-65.

28 Denborough M. Malignant hyperthermia. Lancet 1998; 352: 1131-6.

29 Paul-Pletzer K, Yamamoto T, Bhat MB, Ma J, Ikemoto N, Jimenez LS, et al. Identification of a dantrolene-binding sequence on the skeletal muscle ryanodine receptor. J Biol Chem 2002; 277: 34918-23.

30 Kobayashi S, Bannister ML, Gangopadhyay JP, Hamada T, Parness J, Ikemoto N. Dantrolene stabilizes domain interactions within the ryanodine receptor. J Biol Chem 2005; 280: 6580-7.

31 Kobayashi S, Yano M, Suetomi T, Ono M, Tateishi H, Mochizuki M, et al. Dantrolene, a therapeutic agent for malignant hyperthermia, markedly improves the function of failing cardiomyocytes by stabilizing interdomain interactions within the ryanodine receptor. J Am Coll Cardiol 2009; 53: 1993-2005

32 Szentesi P, Collet C, Sarkozi S, Szegedi C, Jona I, Jacquemond V, et al. Effects of dantrolene on steps of excitation-contraction coupling in mammalian skeletal muscle fibers. J Gen Physiol 2001; 118: 35575.

33 Balam Ortiz EO, Carvajal K, Cruz D. Protective effect of dantrolene in post-ischemic reperfusion myocardial damage. Arch Inst Cardiol Mex 1999; 69: 311-9.

34 Boys JA, Toledo AH, Anaya-Prado R, Lopez-Neblina F, Toledo-Pereyra LH. Effects of dantrolene on ischemia-reperfusion injury in animal 
2007; 404: 431-8

models: a review of outcomes in heart, brain, liver, and kidney. Investig Med 2010; 58: 875-82.

35 Pelleg A, Roth A, Shargordsky B, Belhassen B, Chagnac A, Laniado S. Effects of dantrolene sodium on occlusion and reperfusion arrhythmias in the canine heart. Methods Find Exp Clin Pharmacol 1985; 7: 239-43.

36 Kobayashi S, Yano M, Uchinoumi H, Suetomi T, Susa T, Ono M, et al. Dantrolene, a therapeutic agent for malignant hyperthermia, inhibits catecholaminergic polymorphic ventricular tachycardia in a RyR2(R2474S/+) knock-in mouse model. Circ J 2010; 74: 2579-84.

37 Xu X, Yano M, Uchinoumi H, Hino A, Suetomi T, Ono M, et al. Defective calmodulin binding to the cardiac ryanodine receptor plays a key role in CPVT-associated channel dysfunction. Biochem Biophys Res Commun 2010; 394: 660-6.

38 Meissner A, Min JY, Haake N, Hirt S, Simon R. Dantrolene sodium improves the force-frequency relationship and beta-adregenic responsiveness in failing human myocardium. Eur J Heart Fail 1999; 1: 177-86.

39 Ono M, Yano M, Hino A, Suetomi T, Xu X, Susa T, et al. Dissociation of calmodulin from cardiac ryanodine receptor causes aberrant $\mathrm{Ca}^{2+}$ release in heart failure. Cardiovasc Res 2010; 87: 609-17.

40 Kaneko N. New 1,4-benzothiazepine derivative, K201, demonstrates cardioprotective effects against sudden cardiac cell death and intracellular calcium blocking action. Drug Dev Res 1994; 33: 429 38.

41 Kumagai K, Nakashima H, Gondo N, Saku K. Antiarrhythmic effects of JTV-519, a novel cardioprotective drug, on atrial fibrillation/flutter in a canine sterile pericarditis model. J Cardiovasc Electrophysiol 2003; 14: $880-4$

42 Hachida M, Lu H, Kaneko N, Nonoyama M, Koyanagi H. Protective effect of JTV519 on prolonged myocardial preservation. Transplant Proc 1999; 31: 1094.

43 Inagaki K, Kihara Y, Hayashida W, Izumi T, Iwanaga Y, Yoneda T, et al. Anti-ischemic effect of a novel cardioprotective agent, JTV519, is mediated through specific activation of delta-isoform of protein kinase C in rat ventricular myocardium. Circulation 2000; 101: 797-804.

44 Kimura J, Kawahara M, Sakai E, Yatabe J, Nakanishi H. Effects of a novel cardioprotective drug, JTV-519, on membrane currents of guinea pig ventricular myocytes. Jpn J Pharmacol 1999; 79: 275-81.

45 Nakaya H, Furusawa Y, Ogura T, Tamagawa M, Uemura H. Inhibitory effects of JTV-519, a novel cardioprotective drug, on potassium currents and experimental atrial fibrillation in guinea-pig hearts. $\mathrm{Br} J$ Pharmacol 2000; 131: 1363-72.

46 Yano M, Kobayashi S, Kohno M, Doi M, Tokuhisa T, Okuda S, et al. FKBP12.6-mediated stabilization of calcium-release channel (ryanodine receptor) as a novel therapeutic strategy against heart failure. Circulation 2003; 107: 477-84.

47 Wehrens XH, Lehnart SE, Reiken SR, Deng SX, Vest JA, Cervantes D, et al. Protection from cardiac arrhythmia through ryanodine receptorstabilizing protein calstabin2. Science 2004; 304: 292-6.

48 Kohno M, Yano M, Kobayashi S, Doi M, Oda T, Tokuhisa T, et al. A new cardioprotective agent, JTV519, improves defective channel gating of ryanodine receptor in heart failure. Am J Physiol Heart Circ Physiol 2003; 284: H1035-42.

49 Lehnart SE, Wehrens XH, Laitinen PJ, Reiken SR, Deng SX, Cheng Z, et al. Sudden death in familial polymorphic ventricular tachycardia associated with calcium release channel (ryanodine receptor) leak. Circulation 2004; 109: 3208-14.

50 Hunt DJ, Jones PP, Wang R, Chen W, Bolstad J, Chen K, et al. K201 (JTV519) suppresses spontaneous $\mathrm{Ca}^{2+}$ release and $\left[{ }^{3} \mathrm{H}\right]$ ryanodine binding to RyR2 irrespective of FKBP12.6 association. Biochem J
51 Liu N, Colombi B, Memmi M, Zissimopoulos S, Rizzi N, Negri S, et al. Arrhythmogenesis in catecholaminergic polymorphic ventricular tachycardia: insights from a RyR2 R4496C knock-in mouse model. Circ Res 2006; 99: 292-8.

52 Sedej S, Heinzel FR, Walther S, Dybkova N, Wakula P, Groborz J, et al. $\mathrm{Na}^{+}$-dependent SR Ca${ }^{2+}$ overload induces arrhythmogenic events in mouse cardiomyocytes with a human CPVT mutation. Cardiovasc Res 2010; 87: 50-9.

53 Yamamoto T, Yano M, Xu X, Uchinoumi H, Tateishi H, Mochizuki M, et al. Identification of target domains of the cardiac ryanodine receptor to correct channel disorder in failing hearts. Circulation 2008; 117 : 762-72.

54 Lehnart SE, Mongillo M, Bellinger A, Lindegger N, Chen BX, Hsueh $W$, et al. Leaky $\mathrm{Ca}^{2+}$ release channel/ryanodine receptor 2 causes seizures and sudden cardiac death in mice. J Clin Invest 2008; 118: 2230-45.

55 Bellinger AM, Reiken S, Dura M, Murphy PW, Deng SX, Landry DW, et al. Remodeling of ryanodine receptor complex causes "leaky" channels: a molecular mechanism for decreased exercise capacity. Proc Natl Acad Sci U S A 2008; 105: 2198-202.

56 Fauconnier J, Thireau J, Reiken S, Cassan C, Richard S, Matecki S, et al. Leaky RyR2 trigger ventricular arrhythmias in Duchenne muscular dystrophy. Proc Natl Acad Sci U S A 2010; 107: 1559-64.

57 Shan J, Betzenhauser MJ, Kushnir A, Reiken S, Meli AC, Wronska A, et al. Role of chronic ryanodine receptor phosphorylation in heart failure and beta-adrenergic receptor blockade in mice. J Clin Invest 2010; 120: 4375-87.

58 Banitt EH, Bronn WR, Coyne WE, Schmid JR. Antiarrhythmics. 2. Synthesis and antiarrhythmic activity of $\mathrm{N}$-(piperidylalkyl)trifluoroethoxybenzamides. J Med Chem 1977; 20: 821-6.

59 Anderson JL, Stewart JR, Perry BA, Van Hamersveld DD, Johnson TA, Conard GJ, et al. Oral flecainide acetate for the treatment of ventricular arrhythmias. N Engl J Med 1981; 305: 473-7.

60 Anderson JL, Gilbert EM, Alpert BL, Henthorn RW, Waldo AL, Bhandari AK, et al. Prevention of symptomatic recurrences of paroxysmal atrial fibrillation in patients initially tolerating antiarrhythmic therapy. A multicenter, double-blind, crossover study of flecainide and placebo with transtelephonic monitoring. Flecainide Supraventricular Tachycardia Study Group. Circulation 1989; 80: 1557-70.

61 Muhiddin K, Nathan AW, Hellestrand KJ, Banim SO, Camm AJ. Ventricular tachycardia associated with flecainide. Lancet 1982; 2: 1220-1.

62 Preliminary report: effect of encainide and flecainide on mortality in a randomized trial of arrhythmia suppression after myocardial infarction. The Cardiac Arrhythmia Suppression Trial (CAST) Investigators. N Engl J Med 1989; 321: 406-12.

63 Watanabe H, Chopra N, Laver D, Hwang HS, Davies SS, Roach DE, et al. Flecainide prevents catecholaminergic polymorphic ventricular tachycardia in mice and humans. Nat Med 2009; 15: 380-3.

64 Hilliard FA, Steele DS, Laver D, Yang Z, Le Marchand SJ, Chopra N, et al. Flecainide inhibits arrhythmogenic $\mathrm{Ca}^{2+}$ waves by open state block of ryanodine receptor $\mathrm{Ca}^{2+}$ release channels and reduction of $\mathrm{Ca}^{2+}$ spark mass. J Mol Cell Cardiol 2010; 48: 293-301.

65 Kang G, Giovannone SF, Liu N, Liu FY, Zhang J, Priori SG, et al. Purkinje cells from RyR2 mutant mice are highly arrhythmogenic but responsive to targeted therapy. Circ Res 2010; 107: 512-9.

66 Bellinger AM, Reiken S, Carlson C, Mongillo M, Liu X, Rothman L, et al. Hypernitrosylated ryanodine receptor calcium release channels are leaky in dystrophic muscle. Nat Med 2009; 15: 325-30.

67 Gonzalez DR, Treuer AV, Castellanos J, Dulce RA, Hare JM. Impaired 
S-nitrosylation of the ryanodine receptor caused by xanthine oxidase activity contributes to calcium leak in heart failure. J Biol Chem 2010; 285: 28938-45.

68 Ullrich ND, Fanchaouy M, Gusev K, Shirokova N, Niggli E. Hypersensitivity of excitation-contraction coupling in dystrophic cardiomyocytes. Am J Physiol Heart Circ Physiol 2009; 297: H1992-2003.

69 Erickson JR, Joiner ML, Guan X, Kutschke W, Yang J, Oddis CV, et al. A dynamic pathway for calcium-independent activation of CaMKII by methionine oxidation. Cell 2008; 133: 462-74.

70 Sag CM, Wadsack DP, Khabbazzadeh S, Abesser M, Grefe C, Neumann K, et al. Calcium/calmodulin-dependent protein kinase II contributes to cardiac arrhythmogenesis in heart failure. Circ Heart Fail 2009; 2: 664-75.

71 Erickson JR, Anderson ME. CaMKII and its role in cardiac arrhythmia. J Cardiovasc Electrophysiol 2008; 19: 1332-6.

72 Lehnart SE, Wehrens XH, Reiken S, Warrier S, Belevych AE, Harvey
$\mathrm{RD}$, et al. Phosphodiesterase 4D deficiency in the ryanodine-receptor complex promotes heart failure and arrhythmias. Cell 2005; 123 : 25-35.

73 Reiken S, Wehrens XH, Vest JA, Barbone A, Klotz S, Mancini D, et al. Beta-blockers restore calcium release channel function and improve cardiac muscle performance in human heart failure. Circulation 2003; 107: 2459-66.

74 Poole-Wilson PA, Swedberg K, Cleland JG, Di Lenarda A, Hanrath P, Komajda $\mathrm{M}$, et al. Comparison of carvedilol and metoprolol on clinical outcomes in patients with chronic heart failure in the Carvedilol Or Metoprolol European Trial (COMET): randomised controlled trial. Lancet 2003; 362: 7-13.

75 Wehrens XH, Lehnart SE, Reiken S, Vest JA, Wronska A, Marks AR. Ryanodine receptor/calcium release channel PKA phosphorylation: a critical mediator of heart failure progression. Proc Natl Acad Sci U S A 2006; 103: 511-8. 